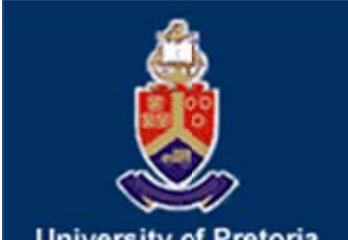

University of Pretoria Department of Economics Working Paper Series

\title{
Perturbed Utility and General Equilibrium Analysis
}

Wei Ma

Xi' an Jiaotong-Liverpool University and University of Pretoria

Working Paper: 2017-01

January 2017

Department of Economics

University of Pretoria

0002, Pretoria

South Africa

Tel: +27 124202413 


\title{
Perturbed Utility and General Equilibrium Analysis
}

\author{
Wei Ma*
}

\begin{abstract}
We study general equilibrium theory of complete markets in an otherwise standard economy with each household having an additive perturbed utility function. Since this function represents a type of stochastic choice theory, the equilibrium of the corresponding economy is defined to be a price vector that makes its mean expected demand equal its mean endowment. We begin with a study of the economic meaning of this notion, by showing that at any given price vector, there always exists an economy with deterministic utilities whose mean demand is just the mean expected demand of our economy with additive perturbed utilities. We then show the existence of equilibrium, its Pareto inefficiency, and the upper hemi-continuity of the equilibrium set correspondence. Specializing to the case of regular economies, we finally demonstrate that almost every economy is regular and the equilibrium set correspondence in this regular case is continuous and locally constant.
\end{abstract}

Keywords: General equilibrium; Stochastic choice; Regular economy

\section{Introduction}

The classical theory of general equilibrium, as initiated in Walras (2003) and culminated in Debreu (1959), postulates that an individual preference be deterministic. The standing of this postulate has been submitted to test by a number of empirical studies, as for instance Davidson and Marschak (1959), and the result has cast grave doubt on its standing. This leads Block and Marschak (1960, p. 97) to remark that "there is a need to substitute 'stochastic consistency of choices' for 'absolute consistency of choices."'We are then confronted with two questions: First, how to construct a utility theory that can best represent the stochastic consistency and, second, under this new theory, in what sense and to what extent we can reconstitute the classical general equilibrium theory.

\footnotetext{
*International Business School Suzhou, Xi' an Jiaotong-Liverpool University, China.

Department of Economics, University of Pretoria, Pretoria, South Africa.
} 
For the first question a multitude of attacks have been made, beginning with Thurstone (1927) through Marschak's work mentioned above to more recent work of Fudenberg et al. (2015) and Gul et al. (2014). In broad outline they fall under two main headings: random utility maximization model and additive perturbed utility (APU) model, with all but Fudenberg et al. (2015) belonging to the first class. These two types of models have overlap but neither nests the other.

As regards the second question the theory of general equilibrium under random utility maximization model has been studied with the pioneering work of Hildenbrand (1971). The main objective here is to examine the statistical properties of the total excess demand and of the equilibrium price vectors (cf. Bhattacharya and Majumdar (1973)). A typical result along this line of research is exemplified by a kind of a central limit theorem: The total excess demand per capita of a random economy tends, under suitable conditions, to be normally distributed when the number of households in it increases without limit.

With this background the present paper undertakes to investigate general equilibrium theory under the APU model of Fudenberg et al. (2015). Our objective is to reconstitute as many aspects as possible of the classical general equilibrium theory. More specifically we shall examine what is an appropriate notion of equilibrium, its existence, efficiency, determinacy, and the properties of the equilibrium set correspondence. The major contrast with the research referred to in the last paragraph is that the total excess demand no longer forms a random variable and there is accordingly no way to study its statistical properties; but instead APU provides us with a possibility to study, under stochastic choice theory, non-convex economies and the efficiency of their equilibria. In fact, the implicit theme underlying the current investigation is that randomization supplies to us a means to handle in a satisfactory manner non-convex economies: it helps to restore some results that fail to hold for non-convex economies with deterministic utilities (cf. Section 4).

We begin in Section 2 with a review of the APU model. Since it applies only to simple lotteries (i.e. lotteries with finite support), the proper framework for our purpose is Mas-Colell (1977a)'s model of indivisible commodities. Specifically we assume all commodities are indivisible but one, which guarantees that there is, at any strictly positive price vector, only finitely many feasible points on the budget line, and focus our attention on economies with a continuum of households. Let $\mathbb{X}$ be the corresponding consumption space and $\mathcal{M}(\mathbb{X})$ the set of simple lotteries on it. For any $\phi \in \mathcal{M}(\mathbb{X})$ which has $\left\{x^{1}, \ldots, x^{n}\right\}$ as its support and $\phi_{i}$ as the probability of $x^{i}$, APU measures the utility of $\phi$ in accordance with

$$
U(\phi)=\sum_{i=1}^{n} u\left(x^{i}\right) \phi_{i}-c\left(\phi_{i}\right)
$$

where $u$ is a function on $\mathbb{X}$ and $c$ one on $[0,1]$. Observing that APU is not continuous with respect to the weak topology on $\mathcal{M}(\mathbb{X})$, we set out to establish a different one such that the desired continuity obtains, and show $\mathcal{M}(\mathbb{X})$ endowed with the new topology is separable and locally compact. We continue with a study of the continuity of the budget set correspondence and the 'demand function,' and close Section 2 with a definition of an allocation as well as its feasibility and Pareto efficiency.

In Section 3 we define the notion of equilibrium for an economy $E$, namely, as a price vector that makes the mean (or total) expected demand of $E$ equal its mean endowment. We begin with a study of the economic meaning of this notion, by showing that at any given price vector, there always exists an economy with deterministic utilities whose mean demand is just the mean expected 
demand of $E$. The difference with the model of Hildenbrand (1971) lies in the fact that the resultant deterministic economy varies with the given price vector. We then show that an equilibrium exists under favorable conditions but is nevertheless not Pareto efficient in general. We end up the section with a result on the upper hemi-continuity of the equilibrium set correspondence.

Aiming to study the determinacy of equilibria we proceed in Section 4 to examine a special type of economies, i.e. regular economies, of which the definition is the same as in the case of deterministic utilities. By analogy with the latter case we study a set of economies which share the same utility structure but differ in endowment structure. We first set up a measure on the set of all admissible initial endowments and then show that almost every economy is regular. We conclude the section and also the paper by establishing, in the circumstance of regular economies, the continuity of the equilibrium set correspondence and its local constancy. This result is interesting by noting that no geometric restriction is placed on $u$, i.e. it is not required to be concave. Without this requirement the result, as has been proved by Mas-Colell (1977b), would fail to hold for economies with deterministic utilities.

\section{The Model}

We assume given $l(\geq 2)$ commodities on the market with only one of them perfectly divisible and all the others indivisible. Since the divisible commodity is usually taken to be money, it is economically reasonable to assume the demand of the indivisible commodities does not vanish. Let

$$
\mathbb{Z}_{+}^{l-1}=\left\{\left(z_{1}, \ldots, z_{l-1}\right): z_{i} \text { is an integer for every } i \text { and } z_{j}>0 \text { for some } j\right\} ;
$$

then we can take as the consumption space $\mathbb{X}=\mathbb{Z}_{+}^{l-1} \times \mathbb{R}_{+}$, wherein $\mathbb{R}_{+}$stands for the set of nonnegative real numbers. On the other hand we take $\mathbb{R}_{++}^{l}$ as the space of initial endowments. This contrast with the consumption space, of which use will be made in Section 4, makes economic sense and can be understood from the viewpoint of aggregation across goods (cf. Varian (1992, Section 9.3)). Take the car market for instance. It is sometimes convenient and reasonable to model the household's choice of a car without distinguishing whether the car is new or old or what brand it is. Suppose the "average price" of the car is $p$. Then the value of the endowment of a household with a new car in his hand will be above $p$, and that of a household with a used car below $p$. This provides the justification for taking $\mathbb{R}_{++}^{l}$ as the space of initial endowments.

Throughout the paper we shall use $\|\cdot\|$ to denote the 1 -norm of a vector. Let $\mathbb{P}$ be the price simplex, i.e.

$$
\mathbb{P}=\left\{p \in \mathbb{R}_{+}^{l}:\|p\|=1\right\},
$$

and $\mathbb{P}_{++}$its relative interior, $\partial \mathbb{P}$ its boundary. Recall that by a preference being incomplete we understand the existence of at least one pair of commodity bundles that is incomparable to each other. In this paper we assume the households involved all have incomplete but strongly monotone preference on $\mathbb{X}$ (see von Neumann and Morgenstern (1947, pp. 28-29) and Shapley and Baucells (1998) for the reasons a household may possibly have incomplete preference). This means that at a given price level $p \in \mathbb{P}$, the household with initial endowments $e$ will choose from

$$
B(p)=\{x \in \mathbb{X}: p x=p e\},
$$

but he may nevertheless not be able to determine the preference relation of $x^{1}$ and $x^{2}$ for some 
$x^{1}, x^{2} \in B(p)$. If, and this will be assumed in the sequel, the household is forced to decide between $x^{1}$ and $x^{2}$, we postulate that he will do so in a random fashion.

To describe this random behavior let $\mathcal{M}(\mathbb{X})$ be the set of simple lotteries on $\mathbb{X}$, i.e. probability measures with a finite support. Noting that $B(p)$ is a finite set on $\mathbb{P}_{++}$, the household's choice among $B(p)$ can therefore be formalized by an element of $\mathcal{M}(\mathbb{X})$. Since a great deal of notation will be introduced presently, it seem appropriate here to make a convention on the symbolism: we shall use $\phi, \phi^{1}, \phi^{2}$ and the like to denote generic elements of $\mathcal{M}(\mathbb{X})$; for $\phi$ and for any other vector that will appear in this paper, we shall use superscripts to distinguish between different vectors and subscripts to indicate the components of a vector.

According to Fudenberg et al. (2015) the random behavior of the household can, as stated in the introduction, be represented by a perturbed utility function $U: \mathcal{M}(\mathbb{X}) \rightarrow \mathbb{R}$, assuming the form

$$
U(\phi)=\sum_{i=1}^{n} u\left(x^{i}\right) \phi_{i}-c\left(\phi_{i}\right),
$$

such that $\phi^{1}$ is preferred to $\phi^{2}$ if, and only if, $U\left(\phi^{1}\right) \geq U\left(\phi^{2}\right)$. Here $\phi \in \mathcal{M}(\mathbb{X})$ has as its support $\left\{x^{1}, \ldots, x^{n}\right\}$ and $\phi_{i}$ the probability of $x^{i} ; u: \mathbb{X} \rightarrow \mathbb{R}$ is continuous and strictly increasing, and $c$ is strictly convex on $[0,1]$ and continuously differentiable on $(0,1)$. Since $U$ is defined only for simple lotteries (which, as noted above, would be the case for $B(p)$ if $p \in \mathbb{P}_{++}$), we assume

$$
\lim _{\|x\| \rightarrow \infty} u(x)=+\infty \text {. }
$$

This condition (together with others) will make the equilibrium price vector (to be defined later on) a member of $\mathbb{P}_{++}$. Let $\mathcal{U}$ be the set of all such $U$ that fulfill the requirements above.

We now define the notion of an economy under perturbed utility. To this end we need a topology on $\mathcal{U}$. By analog with case of deterministic utility, we identify each $U$ with the set

$$
\left\{\left(\phi^{1}, \phi^{2}\right): U\left(\phi^{1}\right) \geq U\left(\phi^{2}\right)\right\},
$$

and impose on $\mathcal{U}$ the topology of closed convergence. To make this precise however we demand a topology on $\mathcal{M}(\mathbb{X})$ : The usual way is to take the weak topology, but it is not a natural one in the current context because $U$ is not continuous with respect to it. To see this take $l=2$ and $\phi$ to be the Dirac measure at $(1,1)$. Let $\phi^{n}$ be the measure with two-element support $\{(1-1 / n, 1-1 / n),(1+$ $1 / n, 1+1 / n)\}$, each with equal probability. Then as is easily verified, $\phi^{n}$ converges weakly to $\phi$, but, due to the strict convexity of $c, U\left(\phi^{n}\right) \nrightarrow U(\phi)$.

In addition to the continuity of $U$ we require for later considerations the topology on $\mathcal{M}(\mathbb{X})$ to be such as makes $\mathcal{M}(\mathbb{X})$ locally compact, so that the closed-convergence topology on $\mathcal{U}$ is metrizable, and makes the solution to Program (2.5) continuous with respect to the closed-convergence topology on $\mathcal{U}$.

With these requirements in mind we observe that every point of $\mathcal{M}(\mathbb{X})$ can be viewed as an element of $\Omega=\mathbb{R}^{l+1} \times \mathbb{R}^{\infty}$, the space of matrices with $(l+1)$ rows and an infinite number of columns. For example, take $l=2$ and $\phi$ to be the lottery that yields both $(1,2)$ and $(2,1)$ with equal probability. Then we can formulate this measure in terms of a matrix:

$$
\left[\begin{array}{cccc}
1 & 2 & 0 & \cdots \\
2 & 1 & 0 & \cdots \\
0.5 & 0.5 & 0 & \cdots
\end{array}\right] .
$$


To make this correspondence definite we put the lexicographic order, denoted $\leqq_{L}$, on $\mathbb{X}$ : For $x^{i}=$ $\left(x_{1}^{i}, \ldots, x_{l}^{i}\right), i=1,2$,

$$
x^{1} \leqq_{L} x^{2} \text { if and only if } x_{1}^{1} \leq x_{1}^{2} \text { or } x_{s}^{1}=x_{s}^{2}, x_{t}^{1} \leq x_{t}^{2}, 1 \leq s \leq t-1,2 \leq t \leq l .
$$

Throughout the paper we shall make the convention that the elements of any subset of $\mathbb{X}$ are arranged in ascending lexicographical order. For any $\phi$ with support $\left\{x^{1}, \ldots, x^{n}\right\}$ and probability $\phi_{i}$ of taking $x^{i}$ we construct the corresponding matrix by putting $\left(x^{1}, \phi_{1}\right)$ in the first column, $\left(x^{2}, \phi_{2}\right)$ the second, up to $\left(x^{n}, \phi_{n}\right)$, and setting the rest of the columns to be zero; more precisely, with $\phi$ we associate this matrix

$$
\omega(\phi)=\left[\begin{array}{lllll}
x^{1} & \cdots & x^{n} & 0 & \cdots \\
\phi_{1} & \cdots & \phi_{n} & 0 & \cdots
\end{array}\right] .
$$

In the following pages we shall identify $\phi$ with $\omega(\phi)$. On $\Omega$ we place the 1 -norm $\|\cdot\|$ : For any $\omega=\left(\omega_{i j}\right) \in \Omega$ let

$$
\|\omega\|=\max _{1 \leq j \leq \infty} \sum_{i=1}^{l+1}\left|\omega_{i j}\right| .
$$

We first study the property of this norm, then its effect on the topology of $\mathcal{M}(\mathbb{X})$. Take from $\mathcal{M}(\mathbb{X})$ a sequence $\phi^{k}$ which has as its support $\left\{x^{k, 1}, \ldots, x^{k, N_{k}}\right\}$ and has $q_{i}^{k}$ as the probability of $x^{k, i}$, $k=0,1, \ldots$. Then we have

LEMMA 2.1. (i) $\phi^{k} \rightarrow \phi^{0}$ when and only when $N_{k}=N_{0}$ for $k$ sufficiently large and $x^{k, i} \rightarrow x^{0, i}$, $q_{i}^{k} \rightarrow q_{i}^{0}$ for all $i$.

(ii) $\mathcal{M}(\mathbb{X})$ endowed with the 1 -norm is a separable, locally compact space.

PROOF. Let us begin by proving statement (i). The sufficiency is obvious. For the necessity suppose $\phi^{k} \rightarrow \phi^{0}$. Recalling from equation (2.1) the structure of $\mathbb{Z}^{l-1}$ we have $\|x\| \geq 1$ for every $x \in \mathbb{X}$. This together with the convergence of $\phi^{k}$, implies $N_{k}=N_{0}$ for $k$ sufficiently large. And then the convergence of $x^{k, i}$ and $q_{i}^{k}$ is simply a matter of the definition of 1-norm.

For statement (ii) we begin with the separability of $\mathcal{M}(\mathbb{X})$. Let $\mathbb{X}_{0}$ be the set of points in $\mathbb{X}$ whose components are all rational numbers, so that $\mathbb{X}_{0}$ is countable. Let $\mathbb{X}_{0}=\left\{\theta^{1}, \theta^{2}, \ldots\right\}$ and

$$
\mathbb{T}_{n}=\left\{\left(q_{1}, \ldots, q_{n}\right): q_{i} \geq 0, \sum q_{i}=1, q_{i} \text { is rational }\right\},
$$

wherein $q_{i}$ stands for the probability of taking $\theta^{i}$. Then it is not hard to check that the union, $\cup_{n=1}^{\infty} \mathbb{T}_{n}$, is a countable, dense subset of $\mathcal{M}(\mathbb{X})$, so that $\mathcal{M}(\mathbb{X})$ is separable.

We now turn to the local compactness of $\mathcal{M}(\mathbb{X})$. For any $\phi \in \mathcal{M}(\mathbb{X})$ consider the closed unit ball centered at $\phi$ :

$$
\mathfrak{B}(\phi)=\left\{\phi^{\prime}:\left\|\omega\left(\phi^{\prime}\right)-\omega(\phi)\right\| \leq 0.5\right\} .
$$

From the structure of $\mathbb{Z}^{l-1}$ (see (2.1)) it follows that the support of any $\phi^{\prime}$ in $\mathfrak{B}(\phi)$ must be of the same cardinality as that of $\phi$, say, equal to $n$. Then $\mathfrak{B}(\phi)$ is a closed and bounded subset of the finite dimensional space $\mathbb{R}^{l+1} \times \mathbb{R}^{n}$, so that $\mathfrak{B}(\phi)$ is compact, and hence $\mathcal{M}(\mathbb{X})$ is locally compact.

Q.E.D

We are now ready to define the notion of an economy. Endow $\mathcal{U}$ with the topology of closed convergence, so that it becomes, according to Lemma 2.1 and Hildenbrand (1974, Theorem 2, p. 19), a compact metrizable space. Endow $\mathbb{X}$ with the usual Euclidean topology and $\mathcal{H}=\mathcal{U} \times \mathbb{X}$ with the product topology, so that $\mathcal{H}$ is a complete and separable metrizable space; a generic element 
of $\mathcal{H}$ we shall call a household and denote by $h=\left(U^{h}, e^{h}\right)$. By an economy $E$ we shall mean the completion of a Borel probability measure on $\mathcal{H}$ such that $\int e^{h} d E(h)$ is finite. The requirement of $E$ being complete here is purely for technical reason.

In the case of deterministic utilities, it is well-known that the non-convexity of $\mathbb{X}$ may lead to the non-existence of equilibrium. The same phenomenon however will occur also for the economies with APU. For this reason, we shall study, as in Yamazaki (1978), an economy $E$ with a dispersed endowment distribution. More precisely, recall that $\mathbb{R}^{l}$ denotes the $l$-dimensional Euclidean space; let $\mathcal{B}\left(\mathbb{R}^{l}\right)$ be its Borel algebra. Then the endowment distribution of $E$, denoted $\mu_{e}$, is the probability measure on $\left(\mathbb{R}^{l}, \mathcal{B}\left(\mathbb{R}^{l}\right)\right)$ such that

$$
\mu_{e}\left(\mathbf{B}_{l}\right)=E\left\{h \in \mathcal{H}: e^{h} \in \mathbf{B}_{l}\right\}, \text { for every } \mathbf{B}_{l} \in \mathcal{B}\left(\mathbb{R}^{l}\right) .
$$

Given $p \in \mathbb{P}_{++}$the resulting wealth distribution, denoted $\mu_{p, e}$, is defined to be the probability measure on $(\mathbb{R}, \mathcal{B}(\mathbb{R}))$ such that

$$
\mu_{p, e}\left(\mathbf{B}_{1}\right)=E\left\{h \in \mathcal{H}: p \cdot e^{h} \in \mathbf{B}_{1}\right\}, \text { for every } \mathbf{B}_{1} \in \mathcal{B}(\mathbb{R}) .
$$

Then $\mu_{e}$ is said to be dispersed if $\mu_{p, e}$ is absolutely continuous with respect to the Lebesgue measure on $\mathbb{R}$ for every $p \in \mathbb{P}_{++}$. For the sake of convenient reference, an economy with a dispersed endowment distribution, we shall call a dispersed economy, and, throughout the paper, by an economy we shall understand a dispersed one, unless otherwise stated.

The motivation for a dispersed economy $E$ is tied to the continuity of the mean excess demand of $E$. To elaborate on this we need the notion of local cheaper points as introduced by Yamazaki (1978): An $x \in \mathbb{X}$ is said to have local cheaper points at a price vector $p \in \mathbb{P}$, if every neighborhood of $x$ contains a point $x^{\prime} \in \mathbb{X}$ such that $p x^{\prime}<p x$. Note that this notion is independent of utility functions, and so Yamazaki's Corollary 1 still holds in the present context. More precisely, let $C_{p}=\{x \in \mathbb{X}: x$ does not have local cheaper points $\}$, and

$$
\mathcal{H}_{p}=\left\{h \in \mathcal{H}: B_{h}(p) \cap C_{p} \neq \varnothing\right\},
$$

where $B_{h}(p)$ is the budget set as defined in equation (2.2) for household $h$ at price vector $p$. Then we have $E\left(\mathcal{H}_{p}\right)=0$. Using this property Yamazaki (1978) proved that, with non-convex consumption spaces, the individual demand correspondence is upper hemi-continuous for almost every household. ${ }^{1}$ It, and this will be useful in a moment, is worthy of notice that in our case $C_{p}$, for $p \in \mathbb{P}_{++}$, is the set of points in $\mathbb{X}$ whose last components vanish.

Yamazaki's result admits an analogue in our setting. More specifically, let $\Delta\left(B_{h}(p)\right)$ be the set of probability measures on $B_{h}(p)$, and let $\phi(h, p)$ be the $U^{h}$-maximizer on $\Delta\left(B_{h}(p)\right)$, that is, $\phi(h, p)$ is the solution to

$$
\begin{array}{cl}
\max & U^{h}(\phi) \\
\text { s.t. } & \phi \in \Delta\left(B_{h}(p)\right) .
\end{array}
$$

As $U^{h}$ is by assumption strictly concave, $\phi(h, p)$ is unique. In the circumstances where the price vector $p$ is our major concern we also write $\phi^{h}(p)$. Before studying the continuity of $\phi(h, p)$ we first investigate the continuity of $B_{h}(p)$, a result of which use will be made frequently in what follows. For this purpose we need a topology on the set of all finite subsets of $\mathbb{X}$. To echo the topology on $\mathcal{M}(\mathbb{X})$ we adopt the following metric: For any two finite subsets, $X^{1}$ and $X^{2}$, of $\mathbb{X}$,

\footnotetext{
${ }^{1}$ Mas-Colell (1977a) obtained the same result for our current consumption space $\mathbb{X}$.
} 
define

$$
d\left(X^{1}, X^{2}\right)=d_{H}\left(X^{1}, X^{2}\right)+\left|\# X^{1}-\# X^{2}\right|,
$$

where $d_{H}$ stands for the Hausdorff metric and \# for the cardinality of a set. One easily verifies that this is indeed a metric. As above we first study the property of the metric, then the continuity of $B_{h}(p)$ with respect to it. Let $X^{k}=\left\{x^{k, 1}, \ldots, x^{k, N_{k}}\right\}, k=0,1,2, \ldots$. Recall that the elements of $X^{k}$ are arranged in ascending lexicographical order. We claim that

LEMMA 2.2. (i) $X^{k} \rightarrow X^{0}$ when and only when $N_{k}=N_{0}$ for $k$ sufficiently large and $x^{k, i} \rightarrow x^{0, i}$ for all $i$.

(ii) The correspondence $B_{h}(p)$ is continuous in $(h, p)$ for every $h \in \mathcal{H} \backslash \mathcal{H}_{p}$ and $p \in \mathbb{P}_{++}$.

PROOF. Since statement (i) is an immediate consequence of the definition of $d$ we shall skip the formal proof.

For statement (ii) take an arbitrary $p \in \mathbb{P}_{++}$and let $\left(h^{k}, p^{k}\right) \rightarrow(h, p)$, where $h^{k}=\left(U^{k}, e^{k}\right)$, $h=(U, e), h \notin \mathcal{H}_{p}, k=1,2, \ldots$. For notational convenience let $B_{k}=B_{h^{k}}\left(p^{k}\right), B=B_{h}(p)$. Suppose $B_{k}=\left\{x^{k, 1}, \ldots, x^{k, N_{k}}\right\}, B=\left\{x^{1}, \ldots, x^{N}\right\}$. According to statement (i) it suffices to show $N_{k}=N$ for $k$ sufficiently large and $x^{k, i} \rightarrow x^{i}$ as $k \rightarrow \infty$ for $i=1, \ldots, N$.

Let us begin with the former. For any $x \in \mathbb{X}$ let $\bar{x} \in \mathbb{Z}^{l-1}$ be the same vector as $x$ but with the last component deleted, and likewise for $\bar{p}$. Since $h \notin \mathcal{H}_{p}$ and $p \in \mathbb{P}_{++}$, it follows that $\bar{p} \bar{x}^{i}<p e$, $i=1, \ldots, N$. As $h^{k} \rightarrow h$ implies $e^{k} \rightarrow e$, we have $\bar{p}^{k} \bar{x}^{i}<p^{k} e^{k}$, hence $N \leq N_{k}$, for $k$ sufficiently large.

To prove $N_{k}=N$, therefore, we assume by way of contradiction that $N_{k}>N$ for infinitely many $k$. Without loss of generality we may assume $N_{k}>N$ for every $k$. This means there exists for every $k$ a $y^{k} \in B_{k}$ such that $\bar{p} \bar{y}^{k}>$ pe. Since the sequence $\left\{e^{k}\right\}$ is convergent, it is bounded, hence the set, $Y=\left\{\bar{y}^{k}: k=1,2, \ldots,\right\}$, is finite (as $\bar{y}^{k} \in \mathbb{Z}^{l-1}$ ). It then follows that there exists a $\bar{y} \in Y$ such that $\bar{y}=\bar{y}^{k}$ for infinitely many $k$, which we assume are $k_{1}, k_{2}, \ldots$ As $\bar{p} \bar{y}>p e$, we have $\bar{p}^{k_{j}} \bar{y}>p^{k_{j}} e^{k_{j}}$ for $j$ sufficiently large. But this contradicts with $y \in B_{k_{j}}$, which therefore proves $N_{k}=N$ for $k$ sufficiently large.

To prove $x^{k, i} \rightarrow x^{i}$ recall the elements of $B_{k}$ and $B$ are arranged in ascending lexicographic order. Since $p x^{k, i}=p e^{k}, p x^{i}=p e$ and $e^{k} \rightarrow e$ it follows that $\bar{x}^{k, i}=\bar{x}^{i}$ for $k$ large enough, hence that $x^{k, i} \rightarrow x^{i}$. This completes the proof.

Q.E.D

With the aid of Lemma 2.2 we can establish the continuity of $\phi(h, p)$ :

LEMMA 2.3. The function $\phi(h, p)$ is continuous in $(h, p)$ for every $h \in \mathcal{H} \backslash \mathcal{H}_{p}$ and $p \in \mathbb{P}_{++}$.

Proof. Again take an arbitrary $p \in \mathbb{P}_{++}$and let $\left(h^{k}, p^{k}\right) \rightarrow(h, p)$, where $h^{k}=\left(U^{k}, e^{k}\right), h=(U, e)$, $h \notin \mathcal{H}_{p}$; let $B_{k}=B_{h^{k}}\left(p^{k}\right), B=B_{h}(p)$. Since $\phi\left(h^{\prime}, p^{\prime}\right)$ is a function of $\left(h^{\prime}, p^{\prime}\right)$, it is sufficient, according to Hildenbrand (1974, Example 1, p. 21), to show its upper hemi-continuity. For notational convenience let $\phi^{k}=\phi\left(h^{k}, p^{k}\right), \phi=\phi(h, p)$. Suppose $\phi^{k} \rightarrow \phi^{0}$; then, according to Hildenbrand (1974, Theorem 1, p. 24), we have but to show $\phi=\phi^{0}$. Combining part (i) of Lemma 2.1 and Lemma 2.2 we know $\phi^{0} \in \Delta(B)$. So it remains to prove $U\left(\phi^{0}\right) \geq U(q)$ for any $q \in \Delta(B)$. Again by Lemma $2.2, B_{k}$ and $B$ have the same cardinality for $k$ large enough, so that $q \in \Delta\left(B_{k}\right)$. This means $U^{k}\left(\phi^{k}\right) \geq U^{k}(q)$, hence $U\left(\phi^{0}\right) \geq U(q)$ as $U^{k} \rightarrow U$. This proves the continuity of $\phi$ in $(h, p)$.

Q.E.D 
To help illuminate the idea of $\phi(h, p)$ let us consider a specific example. Take $c(\phi)=\eta \phi^{2}$ where $\eta>3 / 2$ is a positive scalar. Suppose $B^{h}(p)=\left\{x^{1}, x^{2}, x^{3}\right\}$ and $u\left(x^{i}\right)=i$. Using this information we can solve (2.5) for $\phi(h, p)$, which is a probability measure that produces $x^{1}$ with probability $1 / 3-1 / 2 \eta, x^{2}$ with probability $1 / 3, x^{3}$ with $1 / 3+1 / 2 \eta$. From this we can see that as $\eta$ increases household $h$ will display decreasing selectivity in the sense of Definition 13 of Fudenberg et al. (2015).

To conclude the section we introduce two more notions, those of an allocation and its Pareto efficiency. We define an allocation $\phi$ to be a mapping of $\mathcal{H}$ into $\mathcal{M}(\mathbb{X})$. For notational simplicity let $\phi^{h}=\phi(h)$. Then $\phi$ is said to be feasible for an economy $E$ if

$$
\iint x d \phi^{h}(x) d E(h) \leq \int e^{h} d E(h) .
$$

Since $\int x d \phi^{h}(x)$ can be interpreted as the expected consumption bundle for household $h$, the notion of feasibility requires that the mean expected consumption bundle of an economy should not exceed its mean endowment. A justification for this notion will be given in the ensuing section after the idea of equilibrium has been defined. An allocation $\phi$ is said to be Pareto efficient if it is feasible and there does not exist a feasible allocation $\phi^{\prime}$ such that $U^{h}\left(\phi^{\prime h}\right)>U^{h}\left(\phi^{h}\right)$ for a.e. $h \in \mathcal{H}$.

\section{Equilibrium and Its Properties}

In this section we shall define the notion of an economic equilibrium under perturbed utility and study its existence, Pareto efficiency, and the continuity of the equilibrium set correspondence. Let us start out with the definition of an equilibrium.

\subsection{Definition and Interpretation}

Roughly speaking, an equilibrium price vector for an economy $E$ is one such that the mean of the corresponding expected excess demand is non-positive. More formally, recall $\phi^{h}(p)$ is the $U^{h}$-maximizer on $\Delta\left(B_{h}(p)\right)$; we define the mean expected excess demand of $E$ to be

$$
\Phi_{E}(p)=\iint x d \phi^{h}(p) d E(h)-\int e^{h} d E(h) .
$$

Let $\phi(p)=\left\{\phi^{h}(p): h \in \mathcal{H}\right\}$. Then an equilibrium is defined to be a combination of a price vector $p$ and an allocation $\phi(p)$ such that $\Phi_{E}(p)=0$. Sometimes we write $\Phi(E, p)$ when the economy $E$ is taken also as a variable.

We begin by establishing the meaning of $\Phi_{E}(p)$ and of the corresponding equilibrium, by comparing to the economies with deterministic utilities. According to Agranov and Ortoleva (2015) stochastic choice may arise from incomplete preferences, which in turn may be attributed to the fact that the decision maker, rather than being an individual, is actually a coalition (cf. Baucells

and Shapley (2008)). On this account we understand each household as a coalition and interpret $\Phi_{E}$ from this viewpoint.

First of all, let us consider a concrete example to get some intuition. Assume that each household $h$ consists of three members: father, mother, and child, each of them endowed with a complete (deterministic) preference, denoted respectively by $\succsim_{f}^{h}, \succsim_{m}^{h}, \succsim_{c}^{h}$, and having an equal endowment 
$e^{h}$. Let $\succsim^{h}$ be the preference of household $h$ which is defined as: For any two consumption bundles $x, y$

$$
x \succsim^{h} y \Leftrightarrow x \succsim_{i}^{h} y, \text { for all } i \in\{f, m, c\} .
$$

Assume that every $B_{h}(p)$ contains exactly three elements: $\left\{x^{h, 1}, x^{h, 2}, x^{h, 3}\right\}$ and that

$$
\begin{aligned}
x^{h, 1} & \succsim_{f}^{h} x^{h, 2} \\
x^{h, 2} & \succsim_{f}^{h} x^{h, 3}, \\
x^{h, 1} & \succsim_{m}^{h} x^{h, 3}, \\
x^{h, 3} \succsim_{c}^{h} x^{h, 1} & \succsim_{c}^{h} x^{h, 2},
\end{aligned}
$$

so that household $h$ would be indecisive between $x^{h, 1}, x^{h, 2}, x^{h, 3}$. Assume the household will encounter the situation infinitely many times and each time it is forced to make a decision, in accordance with the following mechanism: one of its three members is picked at random, whose decision will then be taken as that of the household. On this account the demand of the household will be the probability measure $\phi^{h}$ which yields each $x^{h, i}$ with equal probability of $1 / 3, i=1,2,3$. Hence

$$
\iint x d \phi^{h} d E(h)=\frac{1}{3} \int\left(x^{h, 1}+x^{h, 2}+x^{h, 3}\right) d E(h),
$$

from which there follows

$$
\Phi_{E}(p)=\frac{1}{3} \int\left(x^{h, 1}+x^{h, 2}+x^{h, 3}\right) d E(h)-\int e^{h} d E(h) .
$$

So $\Phi_{E}(p)$ can be interpreted as the mean excess demand of the set, $\mathcal{H} \times\{1,2,3\}$, of individuals.

To make this idea precise we introduce some notation. Let $\mathcal{P}$ be the set of preference relations on $\mathbb{X} \times \mathbb{X}$, i.e. preorders that are complete, transitive, and continuous. Endow $\mathcal{P}$ with the topology of closed convergence and $\mathcal{P} \times \mathbb{X}$ with the product topology (remember that $\mathbb{X}$ is equipped with the Euclidean topology). We define a deterministic economy to be a Borel probability measure on $\mathcal{P} \times \mathbb{X}$. Our objective is to find a deterministic economy whose mean excess demand is exactly the mean expected excess demand of the economy $E$.

For this purpose we have to idealize the situation somewhat by assuming that every household contain a continuum of individuals. Let $T=[0,1]$ denote that continuum and by $(h, t)$ we shall mean individual $t$ in household $h$. Let $B_{h}(p)=\left\{x^{h, 1}(p), \ldots, x^{h, N_{h}}(p)\right\}$ for $p \in \mathbb{P}_{++}$. The information at our disposal is the proportion, say $\tau^{h, n}$, of individuals in $T$ who have $x^{h, n}(p)$ as their demanded bundle. It is, however, not easy at all to specify for every individual a demand function $D_{h t}(p)$ such that the Lebesgue measure of the set, $\left\{t \in T: D_{h t}(p)=x^{h, n}(p)\right\}$, is equal to $\tau^{h, n}$ and there exists a preference $\succeq^{h t}$ in $\mathcal{P}$ which is maximized by $D_{h t}(p)$ on the budget set $B_{h}(p)$.

In view of this we retreat somewhat by permitting each individual's preference to vary with $p$ but to be fixed on $B_{h}(p)$. Then we have the following:

LEMMA 3.1. For every $x \in B_{h}(p)$ there exists such a preference in $\mathcal{P}$ as is maximized at $x$.

PROOF. Let $c_{i}=p_{i} x_{i} / p e^{h}$, where $p_{i}, x_{i}$ are the $i$ th components of $p$ and $x$, respectively. Construct the Cobb-Douglas utility function $\sum_{i=1}^{l} c_{i} \ln y_{i}$; it is readily verified that this function has $x$ as its maximizer on $B_{h}(p)$.

Q.E.D

With the aid of this lemma we can prove the main result of this section:

THEOREM 3.1. For every $p \in \mathbb{P}_{++}$there exists a deterministic economy which has $\Phi_{E}(p)$ as its mean excess demand function. 
ProOF. Fix some $p \in \mathbb{P}_{++}$; let $B_{h}(p)=\left\{x^{h, 1}(p), \ldots, x^{h, N_{h}}(p)\right\}$ and recall that $\phi(h, p)$ is the maximizer of $U^{h}$ on $\Delta\left(B_{h}(p)\right)$. Since $p$ is fixed, we shall for the sake of notational convenience suppress the argument $p$ in the expressions $B_{h}(p), \phi(h, p)$ and the like, and write them simply as $B_{h}, \phi^{h}$, etc. Divide $T$ into $N_{h}$ subintervals, $T^{h, 1}, \ldots, T^{h, N_{h}}$, such that $\lambda\left(T^{h, n}\right)=\phi^{h}\left(x^{h, n}\right)$, where $\lambda$ denotes the Lebesgue measure on $\mathbb{R}$. According to Lemma 3.1 we can find a preference $\succcurlyeq^{h, n}$ which has $x^{h, n}$ as its maximizer on $B_{h}$. Now define a mapping $f$ from $\mathcal{H} \times T$ to $\mathcal{P} \times \mathbb{X}$ :

$$
f(h, t)=\left(\succcurlyeq^{h, n}, e^{h}\right) \text { for } t \in T^{h, n} .
$$

Endow $\mathcal{H} \times T$ with the product measure and denote its completion by $\pi$. Assume for a moment that $f$ is measurable and define a deterministic economy $\mathfrak{E}_{p}$ as follows: For any $\mathbf{B}$ in the Borel $\sigma$-algebra of $\mathcal{P} \times \mathbb{X}$

$$
\mathfrak{E}_{p}(\mathbf{B})=\pi\{(h, t): f(h, t) \in \mathbf{B}\} .
$$

On account of the measurability of $f$ the economy $\mathfrak{E}_{p}$ is well defined and, as one easily verifies by means of Fubini's theorem, has $\Phi_{E}(p)$ as its mean excess demand function.

So there remains to show the measurability of $f$. For this purpose it suffices to show that $f$ is almost everywhere continuous. This allows us to assume without restriction of generality that every interval $T^{h, n}$ is open; denote its larger endpoint by $\bar{t}^{h, n}$. Let $\bar{T}^{h}=\left\{\bar{t}^{h, 1}, \ldots, \bar{t}^{h, N_{h}}\right\}, \mathcal{T}_{0}=$ $\cup_{h \in \mathcal{H}}\{h\} \times \bar{T}^{h}$, and $\mathcal{T}_{1}=(\mathcal{H} \times T) \backslash \mathcal{T}_{0}$. The technical difficulty with $\mathcal{T}_{0}$ is that its measurability is open. Happily, however, we notice that its every measurable subset, according to Fubini's theorem, is of zero measure, so that $\mathcal{T}_{0}$ is of zero inner measure. This means that $\pi$ can be extended to a larger $\sigma$-algebra such that $\mathcal{T}_{0}$ is measurable and of zero measure. More specifically, let $\mathcal{B}(\mathcal{H} \times T)$ be the $\sigma$-algebra generated by the union of $\mathcal{B}(\mathcal{H}) \otimes \mathcal{B}(T)$ and $\mathcal{T}_{0}$, where $\mathcal{B}(\mathcal{H}) \otimes \mathcal{B}(T)$ is the product $\sigma$-algebra on $\mathcal{H} \times T$; then there exists a measure on $\mathcal{B}(\mathcal{H} \times T)$, denoted by $\bar{\pi}$, such that

$$
\bar{\pi}\left(\mathcal{T}_{0}\right)=0, \bar{\pi}(\mathbf{B})=\pi(\mathbf{B}) \text { for every } \mathbf{B} \in \mathcal{B}(\mathcal{H}) \otimes \mathcal{B}(T) .^{2}
$$

Recall the definition of $\mathcal{H}_{p}$ from equation (2.4); let $\mathcal{T}=\mathcal{T}_{1} \backslash\left(\mathcal{H}_{p} \times T\right)$, so that $\mathcal{T}$ is of full measure. We proceed to show that $f$ is continuous on $\mathcal{T}$. Suppose $\left(h^{k}, t^{k}\right) \rightarrow\left(h^{0}, t^{0}\right) \in \mathcal{T}, k=$ $1,2, \ldots$. Then by Lemma $2.3, \phi\left(h^{k}, p\right) \rightarrow \phi\left(h^{0}, p\right)$, and so referring to Lemma 2.1, we may assume the supports of $\phi\left(h^{k}, p\right)$ all have the same cardinality. Let $\phi\left(h^{k}, p\right)$ has $\left\{x^{k, 1}, \ldots, x^{k, N}\right\}$ as its support with $q_{i}^{k}$ of taking $x^{k, i}, k=0,1, \ldots$; then $x^{k, i} \rightarrow x^{0, i}$ and $q_{i}^{k} \rightarrow q_{i}^{0}$ for all $i$. Recalling from the first paragraph of this proof the definition of $T^{h^{k}, i}$ we have

$$
T^{h^{k}, 1}=\left(0, q_{1}^{k}\right) \text { and } T^{h^{k}, i}=\left(\sum_{j=1}^{i-1} q_{i}^{k}, \sum_{j=1}^{i} q_{i}^{k}\right), i=2, \ldots, N ; k=0,1, \ldots
$$

This implies that if $t^{0} \in T^{h^{0}, i}$ then $t^{k} \in T^{h^{k}, i}$, so that we may assume $f\left(h^{k}, t^{k}\right)=\left(\succcurlyeq^{k, i}, e^{k}\right)$ for $k=0,1,2, \ldots$. Since $\succcurlyeq^{k, i}$ has $x^{k, i}$ as its maximizer it follows from the construction of $\succcurlyeq^{k, i}$ (cf. the proof of Lemma 3.1) that $\succcurlyeq^{k, i} \rightarrow \succcurlyeq^{i, 0}$, hence that $f$ is continuous. This completes the proof. Q.E.D

\subsection{Existence and Efficiency}

This subsection will study the existence of equilibrium and its Pareto efficiency. Concerning the first aspect we have

\footnotetext{
${ }^{2}$ This is possible as is seen from http://math.stackexchange.com/questions/1181869/extension-of-measure-onsigma-algebra.
} 
THEOREM 3.2. Every dispersed economy has an equilibrium.

Proof. Let $\Phi(p)$ be the mean expected excess demand for an economy $E$ as defined in equation (3.1). According to Mas-Colell et al. (1995, Proposition 17.C.1, p. 585), it suffices to show that on $\mathbb{P}_{++}, \Phi(p)$ is continuous, homogeneous of degree zero, bounded below, satisfies the Walras' law and and the boundary condition, i.e. $\left\|\Phi\left(p_{n}\right)\right\| \rightarrow \infty$ as $p_{n} \rightarrow p \in \partial \mathbb{P}$. That $\Phi(p)$ is homogeneous of degree zero and satisfies the Walras' law is straightforward. Its boundedness from below follows immediately from the finiteness of the mean endowment.

We begin by showing the continuity of $\Phi(p)$. Take $p^{k} \rightarrow p \in \mathbb{P}_{++}$; the proof depends on Lemma 2.3 and Lebesgue's dominate convergence theorem. More specifically, referring to Lemmata 2.1 and 2.3 , we obtain

$$
f_{k}(h) \rightarrow f(h) \text { for any } h \notin \mathcal{H}_{p}
$$

where we have put $f_{k}(h)=\int x d \phi^{h}\left(p^{k}\right)$ and $f(h)=\int x d \phi^{h}(p)$. Observe that $p^{k} f_{k}(h)=p^{k} e^{h}$, which, in combination with $p \in \mathbb{P}_{++}$, implies $f_{k}(h) \leq \tau\left\|e^{h}\right\|$ for some constant $\tau$. Hence according to Lebesgue's dominate convergence theorem, $\Phi\left(p^{k}\right) \rightarrow \Phi(p)$. This proves the continuity of $\Phi(p)$.

It remains to show the boundary condition. For this take $p^{k} \rightarrow p \in \partial \mathbb{P}$. According to Fatou's lemma, it is sufficient to show $\left\|f_{k}(h)\right\| \rightarrow \infty$ for any $h \notin \mathcal{H}_{p}$. So consider an arbitrary $h \notin \mathcal{H}_{p}$. For notational simplicity let $B_{k}=B_{h}\left(p^{k}\right)$ and $\phi^{k}=\phi\left(h, p^{k}\right)$. It is easily seen that there exists an $x^{k} \in B_{k}$ such that $\left\|x^{k}\right\| \rightarrow \infty$, from which however we cannot derive $\int x d \phi^{k} \rightarrow \infty$, because $\phi^{k}\left(x^{k}\right)$ may possibly tend to zero. Fortunately this would not happen, as can be seen from the first-order condition of Programe (2.5): Recall the functional form of $U$ given in equation (2.3) and let $\theta_{k}$ be the Lagrange multiplier, so that for $x^{i} \in B_{k}$

$$
u\left(x^{i}\right)-\theta_{k}=c^{\prime}\left(\phi^{k}\left(x^{i}\right)\right) .
$$

From this we see intuitively that the larger the $x^{i}$, the larger the $\phi^{k}\left(x^{i}\right)$, as the convexity of $c$ implies the monotonicity of $c^{\prime}$. By the intuition alone however we can not formally deduce $\phi^{k}\left(x^{k}\right) \nrightarrow 0$ because $\theta_{k}$ may possibly increase to $\infty$.

To deal with this problem let

$$
A_{m}=\{x \in \mathbb{X}: u(x) \leq m M\},
$$

where $m$ is an integer and $M$ is an arbitrary positive scalar. Since $u(x) \rightarrow \infty$ as $\|x\| \rightarrow \infty$ it follows that $A_{m}$ is a bounded set for any given $m$, hence that $\left|A_{m} \cap B_{k}\right|$ is finite, where $|\cdot|$, when applying to a set, denotes its cardinality. Noting that $\left|B_{k}\right| \rightarrow \infty$ as $p^{k} \rightarrow p \in \partial \mathbb{P}$, there exists for every $m$ an $N_{m}$ such that

$$
\left|B_{N_{m}} \backslash A_{m}\right|>\left|B_{N_{m}} \cap A_{m}\right|,
$$

where \denotes the set-theoretic substraction. Referring to the definition of $A_{m}$ we have $u\left(x^{i}\right)>$ $m M$, hence $u\left(x^{i}\right)>u\left(x^{j}\right)$ and therefore, by equation (3.2), $\phi^{N_{m}}\left(x^{i}\right)>\phi^{N_{m}}\left(x^{j}\right)$, for any $x^{i} \in B_{N_{m}} \backslash A_{m}$ and any $x^{j} \in A_{m}$. This together with (3.3) implies that $\phi^{N_{m}}\left(B_{N_{m}} \backslash A_{m}\right) \geq 0.5$, hence that

$$
\left\|\int x d \phi^{N_{m}}\right\|=\int\|x\| d \phi^{N_{m}} \geq 0.5 \tau_{m}, \text { where } \tau_{m}=\min _{x \in B_{N_{m}} \backslash A_{m}}\|x\| .
$$

To establish the boundary condition therefore we have only to show $\tau_{m} \rightarrow \infty$. Suppose to the contrary that $\tau_{m} \leq \bar{\tau}$ for every $m$ and some scalar $\bar{\tau}$. This means that there exists for every $m$ an $x^{m} \in B_{N_{m}} \backslash A_{m}$ such that $\left\|x^{m}\right\| \leq \bar{\tau}$, hence that, due to the continuity of $u, u\left(x^{m}\right) \leq m M$ and hence 
$x^{m} \in A_{m}$, for $m$ sufficiently large; a contradiction.

After establishing the existence of equilibrium it is then natural to ask about its Pareto efficiency. Let $(p, \phi)$ be an equilibrium. Observe that if $\phi^{h}(p)$ solves

$$
\max U^{h}(\phi) \quad \text { s.t. } p \int x d \phi \leq p e^{h} .
$$

then one can establish, using the feasibility argument as in the case of deterministic utilities (cf. Varian (1992, p. 326)), that $(p, \phi)$ is Pareto efficient. The feasible set of the above problem however is larger than household $h$ 's budget set $B_{h}(p)$, which indicates that the equilibrium might not be Pareto efficient in general. Indeed a counterexample is not hard to come by.

Specifically let $l=2, u(x)=\exp \left(x_{1}+x_{2}\right)$ for any $x=\left(x_{1}, x_{2}\right) \in \mathbb{X}, c(q)=q^{2}$ for any $q \in[0,1]$. Recall the functional form of $U$ from equation (2.3); we construct an economy wherein all households share the same utility function. Let $\alpha:[0,1] \rightarrow \mathcal{H}$ be a mapping such that $\alpha(t)=\left(U, e^{t}\right)$ where $e^{t}=(1,1+t)$; this enables us to indicate the household by $t$. It is not hard to see that $\alpha$ is measurable and thereby induces in the usual way an economy $E$ on $\mathcal{H}$. We shall show the equilibrium of $E$ is not Pareto efficient. First, according to Theorem 3.2, $E$ has an equilibrium, say $(p, \phi)$ and $p \in \mathcal{P}_{++}$; let $\phi=\left(\phi^{t}\right)$. It then follows that the budget set of $t$ is compact, hence that, due to the continuity of $u$, his utility level $U\left(\phi^{t}\right)$ is bounded. Now consider household $t$; we want to construct for him a sequence of probability measures $\phi^{k} \in \mathcal{M}(\mathbb{X})$ whose utilities tend to $\infty$. Let $\phi^{k}$ yield $(0.5 k, 0.5 k)$ with probability $1 / k$ and $(0.5,0.5)$ with $1-1 / k$. It can be verified without difficulty that

$$
\int x d \phi^{k} \leq e^{t} \text { and } U\left(\phi^{k}\right) \rightarrow \infty,
$$

and so $U\left(\phi^{k}\right)>U\left(\phi^{t}\right)$ for $k$ sufficiently large. Therefore, $(p, \phi)$ is Pareto inefficient.

\subsection{The Equilibrium Set Correspondence}

Given an economy $E$ let $\Pi(E)$ be the set of its equilibrium price vectors. In light of Hildenbrand and Mertens (1972), this subsection undertakes to study the continuity of $\Pi(E)$.

To formalize the question we need a topology on the set $\mathcal{E}$ of all economies. For this we shall employ the routine one which induces the following notion of convergence: $E_{n} \rightarrow E$ means $E_{n}$ weakly converges to $E$ and $\int e^{h} d E_{n} \rightarrow \int e^{h} d E$. It is called $\alpha$-topology by Mas-Colell (1977b). With it we have:

THEOREM 3.3. The correspondence $\Pi: \mathcal{E} \rightarrow \mathbb{P}$ is upper hemicontinuous.

Proof. The idea is to use Hildenbrand (1974, Theorem 1, p. 24). First, $\Pi(E) \neq \varnothing$, from Theorem 3.2. Next, $\Pi$ is compact-valued. In fact, more general than this is true:

LEMMA 3.2. If $E_{k} \rightarrow E, p^{k} \rightarrow p, p^{k} \in \Pi\left(E_{k}\right)$, then $p \in \Pi(E)$.

PROOF. Remember that $\phi(h, q)$ is the solution of Program (2.5) when the prevailing price vector is $q$. From the premise of the lemma follows $p \in \mathbb{P}_{++}$. Recall from equation (2.4) the definition of $\mathcal{H}_{p}$. For $h \notin \mathcal{H}_{p}$ take $h^{k} \rightarrow h$. Then by Lemma 2.3, $\phi\left(h^{k}, p^{k}\right) \rightarrow \phi(h, p)$ and so, by part (i) of Lemma 2.1

$$
f_{k}\left(h^{k}\right) \rightarrow f(h),
$$


where for notational economy we have defined $f_{k}(h)=\int x d \phi\left(h, p^{k}\right), f(h)=\int x d \phi(h, p)$.

Now we show $p \in \Pi(E)$. The method is similar to but much simpler than the one used by Hildenbrand and Mertens (1972), as in our case each household has a unique 'demand function' $\phi(h, p)$. Since $E_{k} \rightarrow E$ and $p^{k} \in \Pi\left(E_{k}\right)$, it follows that

$$
\int f_{k}(h) d E_{k}(h) \rightarrow \int e(h) d E(h) .
$$

Associate $T=[0,1]$ with the Lebesgue measure $\lambda$. Since $\mathcal{H}$ is complete and separable it follows from Skorokhod's theorem (cf. Hildenbrand (1974, pp. 50-51, (37))), that there exist measurable mappings $\alpha_{k}$ and $\alpha$ of $T$ into $\mathcal{H}$ such that $E_{k}=\lambda \circ \alpha_{k}^{-1}$ and $E=\lambda \circ \alpha^{-1}$, and $\alpha_{k}(t) \rightarrow \alpha(t)$ a.e. on $T$. Hence we have by (3.4), $f_{k}\left(\alpha_{k}(t)\right) \rightarrow f(\alpha(t))$ a.e. on $T$, so that by Fatou's lemma and (3.5),

$$
\int f(\alpha(t)) d \lambda(t) \leq \int e(\alpha(t)) d \lambda(t) .
$$

This, together with $p f(\alpha(t))=p e(\alpha(t))$ and $p \in \mathbb{P}_{++}$, implies that the equality must hold in (3.6), which therefore completes the proof.

Q.E.D

From the lemma it follows at once that $\Pi$ is compact-valued. With this the theorem then follows conjointly from the lemma and Hildenbrand's theorem mentioned at the very beginning of the proof.

Q.E.D

\section{Regular Economies}

The notion of regularity of an economy springs from the study of the question as to the uniqueness of equilibria. This section will undertake to study the relative position of regular economies in the set of all economies and then, in light of Dierker (1975) and Mas-Colell (1977b), investigate in this regular case some properties of the equilibrium set correspondence as defined in Section 3.3. Let us begin with the first question.

\subsection{Genericity Analysis}

In the case of finite economies with deterministic utilities the relative position of regular economies is discussed by means of genericity analysis (cf. Mas-Colell et al. (1995, Chapter 17, pp. 593597)). Our aim here is to develop its analogue for the current setup.

Let us recall how it is carried out in the former case. The point of view here is to consider, rather than an individual economy, a space of economies which share the same utility function but differ in their initial endowments, so that each economy can be parametrized by the vector of its initial endowments. We first impose on the utility function some favourable conditions which imply a smooth demand function, and then associating the set of all admissible vectors of initial endowments with the Lebesgue measure, we show that almost every economy is regular. To avoid cross-reference we reproduce here the definition of regularity from Mas-Colell et al. (1995, Section 17.D, p. 591): Recall that $D_{p} \Phi_{E}(p)$ represents the Jacobian matrix of $\Phi_{E}(p)$ with respect to $p$.

DEFINITION 4.1. An equilibrium price vector $p$ for an economy $E$ is regular if $D_{p} \Phi_{E}(p)$ has rank $l-1$. The economy is said to be regular if all of its equilibrium price vectors are regular. 
By analogue with the first aspect concerning the utility function, we shall throughout this section restrict our consideration to $u \in C^{2}(\mathbb{X}), c \in C^{2}[0,1]$, and assume, to avoid corner solutions, the positivity condition of Fudenberg et al. (2015, Definition 1), i.e. the solution of Program (2.5) has none of its components vanishing. At the same time, we confine ourselves to the economies with compact support, a limit whose necessity has been disclosed by Dierker (1975) . These conditions, as will be seen later in Lemma 4.1, suffices to assure us of a continuously differentiable mean expected excess demand function.

We turn now to the second aspect, i.e. to set up a measure on the set of initial endowments such that almost every economy with its endowments in that set is regular. This is much harder that the first aspect because there is a continuum of households, so that, if the vector of initial endowments is still used as a parametrization of the economy, the transversality theorem, ${ }^{3}$ the main tool in genericity analysis, would not be applicable.

To overcome this problem we observe that for any initial endowment function $e^{h}=\left(e_{1}^{h}, \ldots, e_{l}^{h}\right)$, the subset of economies whose endowment functions are of the form $\left(r_{1} e_{1}^{h}, \ldots, r_{l} e_{l}^{h}\right)$ with $r=$ $\left(r_{1}, \ldots, r_{l}\right) \in \mathbb{R}_{++}^{l}$, can be indexed by $r$, and therefore lends itself to genericity analysis. By connecting somehow the results on each of these subsets we can hopefully arrive at the desired result on the entire set of all economies.

To formalize the idea we have to introduce some mathematics. Again let $T=[0,1]$ and associate it with the Lebesgue measure. Since $\mathcal{H}$ is complete and separable it follows from Hildenbrand (1974, p. 50, (37)) that every economy $E$ can be viewed as a measurable mapping of $T$ into $\mathcal{H}$, so that we can interpret each $t \in T$ as a household. Let $e(t)$ be the initial endowment of $t$, then by definition of an economy, $\int e(t) d t<\infty,{ }^{4}$ so that $e$ belongs to the $l$-fold product of the space $L^{1}(T)$ (the space of absolutely integrable functions on $T$ ). This space however, compared with the space $C(T)$ of continuous functions on $T$, is a bit bizarre in behavior, for any of its two points are not distinguishable that differ only on a set of measure zero. More precisely assume for the moment $l=1$. Take $e(t)=1$ for every $t \in T$ and $e^{\prime}(t)=1$ for $t$ irrational, $e^{\prime}(t)=-1$ otherwise. Then $e$ and $e^{\prime}$ are the same from the point of view of $L^{1}(T)$. But it is absurd to have an economy wherein some household has negative endowments. For this reason we shall restrict our consideration to $e(t) \in C^{l}(T)$, the $l$-fold product of $C(T)$. This is fortunately not too serious a restriction as is indicated by the Lusin's theorem. To lighten notation we shall suppress the argument $T$ and write instead $C$ and $C^{l}$.

Let us start out with a review of $C$. It is a separable Banach space when endowed with the norm

$$
\|f\|=\max _{t \in T}|f(t)| \text {. }
$$

Let

$$
\mathbf{B}=\{f \in C:\|f\|=1 \text { and } f(t) \geq 0, \forall t \in T\},
$$

and $\mathbf{B}^{l}$ be its $l$-fold product. Associate $\mathbf{B}^{l}$ with the product topology and place on it a Borel probability measure. Endow $\mathbb{R}_{++}^{l}$ with the Lebesgue measure and the product space $\mathbf{B}^{l} \times \mathbb{R}_{++}^{l}$ with the product measure, to be denoted by $\chi$.

\footnotetext{
${ }^{3}$ This theorem has an infinite-dimensional version. But its validity depends on very demanding conditions, which are not satisfied in the current situation.

${ }^{4}$ Here and hereafter, the integral is in the sense of Lebesgue.
} 
Let $\Gamma$ be the set of all admissible initial endowment functions, that is,

$$
\Gamma=\left\{e: e \in C^{l} \text { and } e(t) \geq 0, \forall t \in T\right\} .
$$

For the sake of convenient reference, an economy whose initial endowment function is in a certain set, we shall call the economy being in that set. For instance, if an economy $E$ has its initial endowment function in $\Gamma$, we shall say that $E$ is in $\Gamma$. Then, and this is the essential point, we can identify $\Gamma$ with $\mathbf{B}^{l} \times \mathbb{R}_{++}^{l}$ in the following fashion: For any two vectors $v_{1}, v_{2} \in \mathbb{R}_{++}^{l}$ let $v^{1} \circ v^{2}$ be their Hadamard product, i.e.

$$
v^{1} \circ v^{2}=\left(v_{1}^{1} v_{1}^{2}, \ldots, v_{l}^{1} v_{l}^{2}\right),
$$

where $v^{i}=\left(v_{1}^{i}, \ldots, v_{l}^{i}\right), i=1,2$. For an endowment function $e$, by $v^{1} \circ e$ (or $e \circ v^{1}$ ) we shall mean a function $e^{\prime}$ of $T$ into $\mathbb{R}_{++}^{l}$ such that $e^{\prime}(t)=v^{1} \circ e(t)$ for $t \in T$. Let $\gamma: \mathbf{B}^{l} \times \mathbb{R}_{++}^{l} \rightarrow \Gamma$ such that

$$
\gamma(e, r)=e \circ r .
$$

Then $\gamma$ is easily seen to be a homeomorphism, and hence $\Gamma$ and $\mathbf{B}^{l} \times \mathbb{R}_{++}^{l}$ are topologically the same, which therefore allows us to impose the measure $\chi$ on $\Gamma$.

With this measure however there is a technical difficulty. Specifically fix an $e \in \mathbf{B}^{l}$ and consider the set $\{e\} \times \mathbb{R}_{++}^{l}$. To this set, as discussed above, we can apply genericity analysis to conclude that there exists a set $\mathcal{R}_{e}$ of full measure such that every economy in $\{e\} \times \mathcal{R}_{e}$ is regular. To reach the desideratum that every economy in $\Gamma_{\text {reg }}=\cup_{e \in \mathbf{B}^{l}}\{e\} \times \mathcal{R}_{e}$ is regular, we have to deal with the measurability of $\Gamma_{\text {reg. }}$. To this end we invoke again the device in the preceding section: Let $\mathcal{F}$ be the product $\sigma$-algebra on $\mathbf{B}^{l} \times \mathbb{R}_{++}^{l}$, and we extend $\chi$ from $\mathcal{F}$ to the $\sigma$-algebra generated by $\mathcal{F} \cup\left\{\Gamma_{\text {reg }}\right\}$ such that $\chi\left(\Gamma_{\text {reg }}\right)=1$, where without danger of confusion we denote the extension still by $\chi$.

With these preparations we can state our main result:

THEOREM 4.1. Almost every economy in $\Gamma$ is regular.

Proof. Take an arbitrary $e \in \mathbf{B}^{l}$ and consider the set $\Gamma_{r}=\left\{e \circ r: r \in \mathbb{R}_{++}^{l}\right\}$. We begin by showing that every economy $E$ in $\Gamma_{r}$ has a continuously differentiable mean expected excess demand function with respect to the price vector $p$ and the vector $r$. Recall equation (3.1) where the mean expected excess demand function is indicated as a function of $p$ alone; here for our purpose we shall write it more explicitly as a function of $(p, r)$. Then we have

LEMMA 4.1. $\Phi_{E}(p, r) \in C^{1}\left(\mathbb{P}_{++} \times \mathbb{R}_{++}^{l}\right)$.

Proof. Take $\left(p^{0}, r^{0}\right) \in \mathbb{P}_{++} \times \mathbb{R}_{++}^{l}$ and let $O$ be its immediate neighborhood. Consider household $t \notin \mathcal{H}_{p}$ where $\mathcal{H}_{p}$ is as defined in equation (2.4); let $B(p, r)$ be his budget set at the price vector $p$ and with his initial endowments given by $e_{r}(t)=e(t) \circ r$. By Lemma 2.2 we may assume the existence, for all $(p, r) \in O$, of an integer $N$ such that $B(p, r)=\left\{x^{1}(p, r), \ldots, x^{N}(p, r)\right\}$. Since $\mathcal{H}_{p}$ is of measure zero and $E$ is assumed to have a compact support, to prove the lemma, it suffices to show $\int x d \phi^{t}(p, r)$ is continuously differentiable, where $\phi^{t}(p, r)$ is the solution to Program (2.5); for which it is in turn sufficient to show the continuous differentiability of $x^{i}$ and $\phi^{t}(p, r)$.

Let us begin with $x^{i}$. Recall that $\bar{x}$ stands for the same vector as $x$ but with its last component deleted; we have again by Lemma 2.2, that $\bar{x}_{i}(p, r)=\bar{x}_{i}\left(p^{\prime}, r^{\prime}\right)$ for any $(p, r),\left(p^{\prime} r^{\prime}\right) \in O$. Denote the last component of $x^{i}(p, r)$ by $x_{L}^{i}(p, r)$ and that of $p$ by $p_{L}$; a straightforward calculation gives

$$
x_{L}^{i}(p, r)=\frac{p e_{r}(t)-\bar{p} \bar{x}^{i}(p, r)}{p_{L}}
$$

whence follows at once the smoothness of $x^{i}(p, r)$ in $(p, r)$. 
We now turn to $\phi^{t}(p, r)$. The idea, as in the case of deterministic utilities, is to apply the classical version of the implicit function theorem. Specifically recall the functional form of $U$ as given in equation (2.3) and form the Lagrangian of Program (2.5):

$$
L=\sum_{i=1}^{N} u\left(x^{i}(p, r)\right) \phi_{i}-c\left(\phi_{i}\right)-\delta\left(\sum \phi_{i}-1\right) .
$$

The first-order conditions are then given by

$$
\begin{aligned}
u\left(x^{i}(p, r)\right)-c^{\prime}\left(\phi_{i}\right)-\delta & =0, i=1, \ldots, N, \\
\sum \phi_{i}-1 & =0 .
\end{aligned}
$$

The Jacobian matrix of this system with respect to $\left(\phi_{1}, \ldots, \phi_{N}, \delta\right)$ is

$$
\left[\begin{array}{ccccc}
-c^{\prime \prime}\left(\phi_{1}\right) & 0 & \cdots & 0 & -1 \\
0 & -c^{\prime \prime}\left(\phi_{2}\right) & \cdots & 0 & -1 \\
\vdots & \vdots & \vdots & \vdots & \vdots \\
0 & 0 & \cdots & -c^{\prime \prime}\left(\phi_{N}\right) & -1 \\
1 & 1 & \cdots & 1 & 0
\end{array}\right] .
$$

Since $c$ is strictly convex it follows that $c^{\prime \prime}(q)>0$ for $q \in(0,1)$, hence the invertibility of the above matrix. Applying the implicit function theorem we conclude that $\phi_{i}$ is continuously differentiable in $\left(x^{1}(p, r), \ldots, x^{N}(p, r)\right)$. This together with the smoothness of $x^{i}$ implies the continuous differentiability of $\phi_{i}$ in $(p, r)$.

Q.E.D

Now according to the transversality theorem (cf., for example, Mas-Colell et al. (1995, Proposition 17.D.3, p. 595)) and the construction of the probability measure $\chi$, to prove the theorem, it remains to show $\operatorname{rank} D_{r} \Phi_{E}(p, r)=l-1$ for any $(p, r) \in \mathbb{P}_{++} \times \mathbb{R}_{++}^{l}$. But this follows from the by now almost standard argument as the one used in Mas-Colell et al. (1995, Proposition 17.D.4, p. 596), and hence the proof is completed.

Q.E.D

\subsection{The Equilibrium Set Correspondence}

Recall that $\Pi(E)$ designates for an economy $E$ the set of its equilibrium price vectors. This subsection seeks to study its continuity and local constancy. In order to avoid complications arising from the null set $\mathcal{H}_{p}$ (cf. equation (2.4)) we take $\mathbb{X}_{++}=\mathbb{Z}^{l-1} \times \mathbb{R}_{++}$as the consumption space. Let $\mathcal{U}_{\text {reg }}$ be the set of functions assuming the form (2.3) with $u \in C^{2}\left(\mathbb{X}_{++}\right)$and $c \in C^{2}(0,1)$. Endow $\mathcal{U}_{\text {reg }}$ with the topology of $C^{2}$ uniform convergence on compact sets, and $\mathcal{H}_{\text {reg }}=\mathcal{U}_{\text {reg }} \times \mathbb{X}_{++}$with the product topology. At the same time endow the set of all subsets of $\mathcal{H}_{\text {reg }}$ with the the Hausdorff metric, and impose on $\mathcal{H}_{\text {reg }}$ what is called by Mas-Colell (1977b) $\beta$-topology: $E_{n} \rightarrow E$ means $E_{n} \rightarrow E$ in the $\alpha$-topology and $\operatorname{supp}\left(E_{n}\right) \rightarrow \operatorname{supp}(E)$.

With these preliminaries we state

THEOREM 4.2. The correspondence $\Pi: \mathcal{E}$ reg $\rightarrow \mathbb{P}$ is continuous, and there exists for every $E \in \mathcal{E}_{\text {reg }}$ a neighborhood $O_{E}$ such that $\# \Pi(E)$ is finite and constant on $O_{E}$.

PROOF. The proof is almost the same as that of Dierker (1975) and hence we shall present an outline only. The basic idea is to use another version of the implicit function theorem (see Appendix). For technical reasons it is convenient to use a different normalization of the price vectors, that is, we set $p_{l}=1$. Also by slight abuse of notation let $\Phi(E, p)$ be the mean expected excess demand of 
the economy $E$ for the first $l-1$ commodities, and let $f(h, p)$ be the vector consisting of the first $l-1$ components of $\int x d \phi(h, p)$ and similarly for $e_{-l}(h)$, so that

$$
\Phi(E, p)=\int f(h, p) d E(h)-\int e_{-l}(h) d E(h) .
$$

From the proof of Theorem 3.3 follows the continuity of $\Phi$ on $\mathcal{E}_{\text {reg }} \times \mathbb{R}_{++}^{l-1}$.

Now we show the continuity of $\partial \Phi(E, p) / \partial p_{i}$ in a neighborhood of $(E, p)$. For this take $E_{k} \rightarrow E$ and $p^{k} \rightarrow p$. Recall from Section 2 that $C_{p}$ is the set of points in $\mathbb{X}$ that do not have local cheaper points. There follows $\mathbb{X}_{++} \cap C_{p}=\varnothing$, and hence $\mathcal{H}_{p}=\varnothing$, for all $p \in \mathbb{R}_{++}^{l-1}$. So the situation becomes all but identical with that of Lemma 3 of Dierker (1975). First, with the aid of the argument of Lemma 4.1, it is not hard although somewhat tedious to verify that as $h^{k} \rightarrow h$ and $p^{k} \rightarrow p \in \mathbb{R}_{++}^{l-1}$,

$$
\frac{\partial f}{\partial p_{i}}\left(h^{k}, p^{k}\right) \rightarrow \frac{\partial f}{\partial p_{i}}(h, p) .
$$

Since $E_{k}$ and $E$ are all assumed to have compact supports, it follows that

$$
\frac{\partial \Phi\left(E_{k}, p^{k}\right)}{\partial p_{i}}=\int \frac{\partial f\left(h^{k}, p^{k}\right)}{\partial p_{i}} d E_{k}(h), \frac{\partial \Phi(E, p)}{\partial p_{i}}=\int \frac{\partial f(h, p)}{\partial p_{i}} d E(h) .
$$

On applying Billingsley (1968, Theorem 5.5, pp. 33-34), which says in our notation that $E_{k} \rightarrow E$ implies $E_{k} f_{k}^{-1}$ converges weakly to $E f^{-1}$, we conclude $\partial \Phi\left(E_{k}, p^{k}\right) / \partial p_{i} \rightarrow \partial \Phi(E, p) / \partial p_{i}$.

Take $E \in \mathcal{E}_{\text {reg }}$ and suppose $\Pi(E)=\left\{q^{1}, \ldots, q^{n}\right\}$. For each $q^{i}$ we know $D_{p} \Phi\left(E, q^{i}\right)$ is invertible. It then follows from the implicit function theorem that there exist neighborhoods $O_{E, i}$ of $E, O_{i}$ of $q^{i}$, and a continuous function $\xi_{i}: O_{E, i} \rightarrow O_{i}$ such that for each $E^{\prime} \in O_{E, i}, \xi_{i}\left(E^{\prime}\right)$ is the unique point in $O_{E, i}$ which satisfies $\Phi\left(E, \xi_{i}(E)\right)=0$. Let $O_{E}=\cap_{i} O_{E, i}$; we have

$$
\Pi\left(E^{\prime}\right)=\cup \xi_{i}\left(E^{\prime}\right) \text { for all } E^{\prime} \in O_{E},
$$

which therefore completes the proof.

Q.E.D

\section{Appendix}

THEOREM. (Implicit function theorem) Let $Z$ be an open subset of $\mathbb{R}^{l}$ and $\Theta$ a metric space; let $f: Z \times \Theta \rightarrow \mathbb{R}^{l}$ be continuous. Suppose the Jacobian matrix $D_{z} f$ of $f$ with respect to $z$ exists at each point $(z, \theta)$ and is continuous on $Z \times \Theta$. Assume $D_{z} f(\bar{z}, \bar{\theta})$ is invertible and $f(\bar{z}, \bar{\theta})=0$. Then there exist neighborhoods $O_{\bar{z}}$ of $\bar{z}, O_{\bar{\theta}}$ of $\bar{\theta}$, and a continuous function $\xi: O_{\bar{\theta}} \rightarrow O_{\bar{z}}$ such that for each $\theta \in O_{\bar{\theta}}, \xi(\theta)$ is the unique point in $O_{\bar{z}}$ which satisfies $f(\xi(\theta), \theta)=0$.

\section{References}

Agranov, M. and Ortoleva, P. (2015). Stochastic choice and preferences for randomization. Working Paper.

Baucells, M. and Shapley, L. S. (2008). Multiperson utility. Games and Economic Behavior, 62(2):329 - 347.

Bhattacharya, R. N. and Majumdar, M. (1973). Random exchange economies. Journal of Economic Theory, 6(1):37 - 67. 
Billingsley, P. (1968). Convergence of probability measures. Wiley.

Block, H. D. and Marschak, J. (1960). Random orderings and stochastic theories of response. Technical Report 66, Cowles Foundation for Research in Economics, Yale University.

Davidson, D. and Marschak, J. (1959). Experimental Tests of a Stochastic Decision Theory, chapter 13, pages 233-269. John Wiley and Sons.

Debreu, G. (1959). Theory of Value: An Axiomatic Analysis of Economic Equilibrium. Yale University Press.

Dierker, H. (1975). Smooth preferences and the regularity of equilibria. Journal of Mathematical Economics, 2(1):43 - 62.

Fudenberg, D., Iijima, R., and Strzalecki, T. (2015). Stochastic choice and revealed perturbed utility. Econometrica, 83(6):2371-2409.

Gul, F., Natenzon, P., and Pesendorfer, W. (2014). Random choice as behavioral optimization. Econometrica, 82:1873-1912.

Hildenbrand, W. (1971). Random preferences and equilibrium analysis. Journal of Economic Theory, 3(4):414 - 429.

Hildenbrand, W. (1974). Core and Equilibria of a Large Economy. Princeton studies in mathematical economics. Princeton University Press.

Hildenbrand, W. and Mertens, J. F. (1972). Upper hemi-continuity of the equilibrium-set correspondence for pure exchange economies. Econometrica, 40(1):99-108.

Mas-Colell, A. (1977a). Indivisible commodities and general equilibrium theory. Journal of Economic Theory, 16(2):443-456.

Mas-Colell, A. (1977b). Regular, nonconvex economies. Econometrica, 45:1387-1407.

Mas-Colell, A., Whinston, M. D., and Green, J. R. (1995). Microeconomic Theory. Oxford University Press.

Shapley, L. S. and Baucells, M. (1998). Multiperson utility. UCLA Economics Working Papers 779.

Thurstone, L. L. (1927). A law of comparative judgment. Psychological Review, 34:273-286.

Varian, H. (1992). Microeconomic Analysis. Norton.

von Neumann, J. and Morgenstern, O. (1947). Theory of Games and Economic Behavior. Princeton Classic Editions. Princeton University Press.

Walras, L. (2003). Elements of Pure Economics: Or the Theory of Social Wealth. Routledge.

Yamazaki, A. (1978). An equilibrium existence theorem without convexity assumptions. Econometrica, 46(3):541-555. 